\title{
A Method for Detection and Modeling of the Human Spine Based on Principal Curvatures
}

\author{
Y. Santiesteban ${ }^{1}$, J.M. Sanchiz ${ }^{2}$, and J.M. Sotoca ${ }^{2}$ \\ ${ }^{1}$ Universidad de Oriente, Santiago de Cuba, Cuba \\ bonsagitaire@yahoo.fr \\ ${ }^{2}$ Universidad Jaume I, Castellón, Spain \\ \{sanchiz, sotoca\}@uji.es
}

\begin{abstract}
The detection and modeling of the human spine from scanned $3 \mathrm{D}$ data is an important issue in biomedical shape analysis. It can be useful for avoiding invasive treatments like radiographs, taken for the purpose of monitoring spine deformations and its correction, as is the cases in scoliosis. This is especially important with children.

This work presents a new method for the detection of the human spine from 3D models of human backs formed by triangular meshes, and taken with a range sensor. The method is based on the estimation of the principal curvatures directions, and by joining valley points along these directions. Results are presented with the method applied to scanned 3D models of real patients.
\end{abstract}

\section{Introduction}

Serious deformities in the human spine are present in a significant percentage of the population, these includes scoliosis, and abnormal cifosis and lordosis. The idiopathic scoliosis is the most common of these deformities, mainly in children [1], this is an abnormal lateral curvature of the spine of congenital origin, or caused by trauma or disease of the vertebrae or hip bones. This is first noticed as a result of the changes that occur in the shape of the human back during the adolescent growing season. The characteristic feature is the disfiguring hump, caused by the rotation of the vertebrae and ribs, that appears together with a lateral bend of the spine.

In some cases the deterioration of the spine occurs quickly, so an early detection of this disease is very important. The most extended means of assessment has been the use of frequent X-ray examinations. Well known is the noxious effect of radiation over patients, principally in early seasons.

Aiming to find alternatives, several non-invasive methods of surface shape measurements and analysis have been proposed and used, including deformation tests [4, photographic methods [2] 3] 5].

Several works have used the analysis of the principal curvatures from 3D data to detect the human spine on the back surface [6] [7. In the present paper, we describe a new method for the detection and modeling of the backbone, which 
constitutes a preliminary step for every method that attempts to estimate the deformation of human spines.

For the development and assessment of our method we have used a 3D representation of the human back, as a computerized 3D model, obtained by a non-invasive technique, such as the use of a laser scanner. These 3D models are triangular meshes where the mesh vertices are real scanned points in the back to be represented.

The structure of this paper is as follows: in Section 2 some related works are discussed. Section 3 describes surface curvature and the approach presented in this paper for estimating these geometric features. In Section 5 we explain our approach in detail. Finally, in Section 7 conclusions are drawn and further research directions are discussed.

\section{Related Work}

The human back presents an almost symmetrical shape, being the spine the symmetry axis. From the lumbar area, in the base of the dorsal thorn, to the cervical region symmetry is preserved. From simple visual inspection of horizontal sections we would be able to locate the whole dorsal thorn estimating their position in each traverse section. The estimation is possible only from the mentioned symmetrical characteristic.

Symmetry of the back is affected by deformations in the spine, mainly due to affections as scoliosis, cifosis or lordosis that produce deviations and torsions. Despite this anomaly it is still possible to find in each horizontal section (profile) the point of best symmetry, that is, the point of minimum asymmetry. This point will, in fact, be a localization of the spine for this horizontal profile. The group of the points in the horizontal profiles will constitute a localization of the dorsal thorn in the all back.

This is the idea followed by Drerup and Hierholzer [6] for the detection of the spine. In their work a fixed coordinate system is attached to the body. The reference points of this reference system were: the prominence of the seventh cervical vertebra, the sacrum point, and the two points dimples of the posterior superior iliac spines. The first two points defined the vertical axis and the others two the horizontal axis. For the localization of reference points the "symmetry line" was detected along the back. Then, by means of anatomical criteria, windows were located where the points of interest were estimated. Inside each region the anatomical points were finally located using digital image processing techniques. These regions were called landmark windows. The authors use as a back surface representation the raster stereographic technique.

Another approach used in order to detect the backbone was presented by Sotoca [8]. In this case the authors use an active shape model 9] based on a statistical scheme trained with a set of samples. This method looked for a curve that represents the shortest path between point matching vertebra $C r$ and point matching vertebra $L 5$. This curve must fulfill also a criterion of surface smoothness lengthwise. This contribution is based on a raster stereographic technique. 


\section{Principal Curvatures Estimation}

The use of invariant geometric properties is very extended in Computer Vision, like principal curvatures and their directions. Surface curvature is a local shape descriptor invariant to rigid body transformations, this is a concept from Differential Geometry [10] 11]. Several works about 3D object analysis and recognition are based on principal curvatures [12 13, 14 [15. Particularly, its use has been adequate in medical imaging applications [16] [17. The use of polyhedral representations for approximation of real surfaces is also quite common. Actually triangular meshes is the most habitual representation for surfaces because of easiness in latter processing such as estimation of geometric features [18. However, estimation of surface curvatures on a discrete graphical model is difficult. The main drawback is that curvatures depend on the second derivative of the surface. Some works aim at overcoming this difficulty; they are grouped regarding the techniques used, and can be classified into three main categories [19. The first one is surface fitting methods, here curvatures are computed from an analytical surface formula that is previously fit to the discrete model points. This approach requires a local surface parameterization 20, 21. The second class is total curvature methods, in this context curvature is estimated for a surface region and not for singles points [22. Finally, curve fitting methods fit a family of curves around each surface point in order to estimate the curvatures 23] 24] 25]. When working with large 3D models, as such the ones present in medical applications, it is very important to use a robust estimation method capable of dealing with noise. In this sense, curve fitting based methods are very promising.

The Normal Vector Voting ( $N V V$ ) algorithm introduced by 19 is an extension of the work presented by [26] and [27]. We have used this method, with slight modifications, for estimating principal curvatures and principal directions. This is a two-pass algorithm that can work with large, and probably noisy, triangular meshes. The first step consists of estimating the normal direction for each surface point. In the second step the principal curvatures and their directions are estimated for each surface point.

Basically, the method uses a voting scheme such that all triangles and vertices in a neighborhood of user defined radium, around the current vertex, affect the estimation of curvatures and directions for this vertex. This approach contrasts with other curvature estimation methods that only use a simple one-ring vicinity around the vertex of interest [28. Interesting in the $N V V$ is that the neighborhood of each vertex is defined using geodesic distance and not with traditional Euclidean distance. The search for the geodesic neighborhood of a vertex is related to the discrete geodesic problem [29]; this concerns about finding a set of triangles within a specified distance to this vertex. Geodesic distance is computed using a modification of the Kimmel and Sethian method [30, introduced by Sun and Abidi in [31].

Having the vicinity, each triangle in this patch (or geodesic neighborhood) votes for the center vertex. These votes are collected in a covariance matrix, 
which is decomposed using eigen-analysis. Then eigenvectors and eigenvalues are used respectively for estimating principal directions and principal curvatures for each point.

We have introduced a slight modification of the $N V V$ algorithm that in our case produce better results, we now explain this fact. When collecting votes, in the first pass of the $N V V$ algorithm for estimating the normal vector orientation of each vertex in the mesh, for each triangle in the geodesic neighborhood of vertex $v$ was defined the matrix $V_{v}=\sum w_{i} V_{i}$. In this definition $V_{i}$ is the covariance matrix $V_{i}=N_{i} N_{i}^{t}$ where $N_{i}$ is the normal vector for each triangle in the geodesic neighborhood. $w_{i}$ is a weighting term defined as $w_{i}=\frac{A_{i}}{A_{\max }} \exp \left(-\frac{g_{i}}{\sigma}\right)$ where $A_{i}$ is the area of each triangle, $g_{i}$ is the geodesic distance from the center of the triangle to the vertex $v, \sigma$ is a constant value defined as function of a maximal geodesic distance defined by the user such that votes from triangle beyond this distance have a negligible influence. The other term in this expression is $A_{\max }$ which is defined as the maximal area in the all mesh. However, the dimension of the triangles in a mesh can vary significantly, and this fact can produce adverse numerical effects in neighborhoods with triangles of small area relative to the maximal area. To overcome this drawback we have used, with better results, the local maximal area in the geodesic neighborhood of the current vertex instead of global maximal area.

\section{Method Overview}

The proposed approach proceeds by computing the minimum asymmetry point located on each of several horizontal profiles placed on the back surface. Each of these points is an estimation of the position of the spine, so we can model the backbone by means of this set of minimum asymmetry points. But in our approach the model used is a triangular mesh, and the way of defining a horizontal profile is different that what it would be using other model for the back surface, for example if we use raster stereography. When we set a horizontal cutting plane in order to find intersections with the surface and obtain points forming this horizontal profile, in the case of triangular meshes we can not control the amount of points in this intersection. So, when defining horizontal profiles we can not just take vertices of the triangular mesh belonging to the cutting plane (see Figure 1).

On the other hand, we use directions of principal curvatures as local shape descriptor of the back surface. We assume that surface is oriented such the backbone is almost vertical. To obtain horizontal profiles we set several horizontal cutting planes intercepting the surface uniformly along that vertical axis (Figure 1 a). To define a profile we take into account all vertices belonging to the corresponding cutting plane and also all vertices between this cutting plane and the following one. Then we project all these points over the current cutting plane and we use all these projected points as profile definition.

When projecting $3 \mathrm{D}$ vertices on a cutting plane several of these point can be very close between them. In general, we are not interested in processing all the projected points placed near the same coordinate in the $x$ axis ((Figure 1) b), 


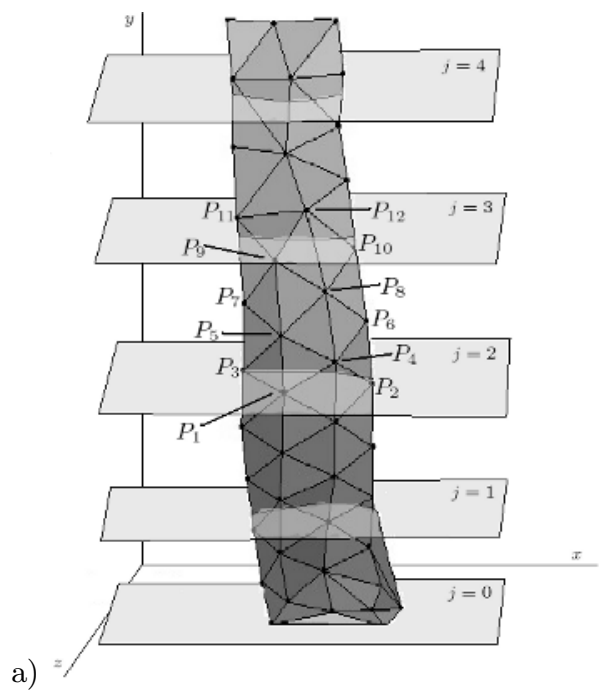

b)

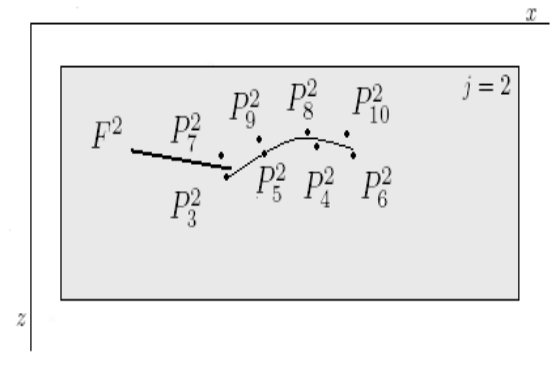

Fig. 1. Horizontal cutting planes defining profiles

regardless coordinate in the $z$ axis. So, we use a clustering method in order to find groups of points close to each other in the cutting plane. Then we use only the central point for each cluster representing all points that have been classified together. This central point is not necessarily a member of the cluster, but the cluster centroid.

So far we have a set of points for each cutting plane, next we need to use a local shape descriptor to analyze the surface behavior in this profile. We select one direction of the principal curvatures for each point as a local shape descriptor. The direction chosen is the most horizontal one. The direction of the curvature for the cluster centroid is obtained from the direction of all points in the cluster.

At this point a profile is formed by a set of points (cluster centroid) and one direction for each of these points. Afterwards we find convex and concave regions taking second derivatives in the profile, then we select one of these regions by a criterion of symmetric position in the profile. The point locating the spine is the middle point of the selected region.

Having a location for the spine on each profile, the entire backbone is modeled by a third degree polynomial. A robust fitting method that can cope with outliers is used to estimate the polynomial parameters.

\section{Human Spine Detection Method Based on Principal Directions}

At this stage we assume that principal curvatures and directions have been estimated for every vertex in the triangular mesh modeling the back surface. In the following we explain in detail how to obtain the horizontal profiles. 
Let $\left\{v_{i}^{1}, v_{i}^{2}\right\}$ be the pair of principal directions for each surface point $P_{i}$, $i=1,2, \ldots, n$, where $n$ is the number of vertices in the mesh. We associate vector $v_{i}$ to each point $P_{i} . v_{i}$ is defined as

$$
v_{i}=\left\{\begin{array}{l}
v_{i}^{1} \text { if }\left|v_{i_{y}}^{1}\right| \leq\left|v_{i_{y}}^{2}\right| \\
v_{i}^{2} \text { other case }
\end{array}\right.
$$

where $v_{i_{y}}^{1}$ and $v_{i_{y}}^{2}$ are the $y$ axis component for the first and second principal directions. In others words, we assign to each point the most horizontal of the two principal curvatures directions.

In the rest of the paper we suppose that the surface orientation and the reference coordinate system is as showed in Figure2. We also assume that points of minimum coordinate in the $y$ axis lie in the $x-z$ plane. So we can define the height of the surface, $L$, as the maximum coordinate in the $y$ axis.

We place several cutting planes intercepting horizontally the surface, and then we project all points over some cutting plane. Denoting as $y=j \lambda$ the family of cutting planes, where $j=0,1,2, \ldots, N, N$ being the number of cutting planes, we can derive that $N=\left[\frac{L}{\lambda}\right], \lambda$ being a constant value representing the spacing between any pair of cutting planes and $[\dot{:}]$ representing the integer part operation. We refer to a cutting plane by its factor $j$, and because there is a single profile for each cutting plane and vice versa, we refer to the profile $j$ which is attached to the cutting plane $j$.

Next we can define how points are projected onto the cutting planes. A point $P\left(x_{P}, y_{P}, z_{P}\right)$ is projected onto cutting plane $j$ if this is the nearest cutting plane, such that $y_{P} \geq j \lambda$. We denote projection of $P$ on plane $j$ as $P^{j}\left(x_{P}, j \lambda, z_{P}\right)$.

In order to simplify the profiles and obtain only useful shape information, we use an incremental clustering technique for grouping close points. Incremental clustering algorithms form clusters dynamically each time a new object is available. These methods are extensively used in document classification, image segmentation and more applications. Given a set of objects to be clustered, clustering algorithms define partitions of this set. For the clustering task a representation space, a similarity measure between objects and a clustering criterion are needed.

In our case, the objects to be grouped are $2 \mathrm{D}$ points $(3 \mathrm{D}$ points projected on a cutting plane). For the clustering algorithm, and as a similarity measure between two point projections $P_{1}^{j}\left(x_{i_{1}}^{j}, j \lambda, z_{i_{1}}^{j}\right)$ and $P_{2}^{j}\left(x_{i_{2}}^{j}, j \lambda, z_{i_{2}}^{j}\right)$ we define the function $s\left(P_{1}^{j}, P_{2}^{j}\right)=\left|x_{i_{1}}-x_{i_{2}}\right|$. This function take as similar points that are close by the $x$ axis component regardless the $z$ axis component.

It is frequent in the literature to use a cluster centroid such that when estimating if new objects will be in a given cluster, comparison is made between the new object and the cluster centroid instead of between the new objects and all objects in the cluster. Each time a new objects is inserted into the cluster, the cluster centroid is updated. We use this approach and we define the cluster centroid for the cluster $C^{j}$ as the point $P_{C^{j}}\left(x_{C^{j}}, j \lambda, z_{C^{j}}\right)$ where $x_{C^{j}}$ and $z_{C^{j}}$ are 
the average coordinate in $x$ and $z$ axis respectively for all points in $C^{j}$. Here $j$ is the cutting plane.

As a clustering criterion we use the fact that $P$ will be in cluster $C^{j}$ if and only if $s\left(P, P_{C^{j}}\right)<r$, where $r$ is a user defined threshold.

We have used the Single-Pass algorithm by Hill 32 for clustering the set of points projected on each cutting plane. This is a simple method that assigns a point to the first cluster that matches the clustering criterion.

Then, we define the set $M^{j}=\left\{P_{C_{1}}^{j}, P_{C_{2}}^{j}, \ldots, P_{C_{m}}^{j}\right\}$ as the set of cluster centroid, here $m$ is the number of clusters for cutting plane $j$. Associated to $M^{j}$, we define the set of directions for each cluster centroid $V^{j}=\left\{v_{1}^{j}, v_{2}^{j}, \ldots, v_{m}^{j}\right\}$. Each vector in $V^{j}$ is obtained by averaging components of the direction vector for each point in the corresponding cluster. We identify the profile for cutting plane $j$ as the set of cluster centroids $M^{j}$, and the set of profile directions is $V^{j}$.

For each profile $j$ we consider the function $f\left(v_{k}^{j}\right)=\tan \alpha_{k}^{j}$, where $\alpha_{k}^{j}$ is the slope angle of $v_{k}^{j}$. All $v_{k}^{j}$ are 2D vectors in cutting plane $j$. Function $f$ has following property: $f\left(v_{k}\right)=\left(F^{j}\right)^{\prime}$, where $F^{j}$ is the analytic intersection curve between the cutting plane and the surface. After that, we consider the function $g\left(v_{k}^{j}\right)=G\left(f\left(v_{k}^{j}\right)\right)$, where $G$ is a first derivative Gaussian function. The use of a Gaussian function has the effect of smoothing the profile and obtaining its second derivative, then we have that $g\left(v_{k}^{j}\right)=\left(F^{j}\right)^{\prime \prime}$. So far profiles have been defined, the next step is the detection of concave and convex regions for each profile. Aiming at this we use the following statement: $P_{C_{k}}^{j}$ is in a concave region if $g\left(v_{k}^{j}\right)>0$. On the other hand, if $g\left(v_{k}^{j}\right)<0$ then $P_{C_{k}}^{j}$ is on a convex region.

In this manner, our method selects points from concave and convex regions for each profile. By means of a symmetry criterion in the profile, using the geodesic distance, we select only one point for each profile. At the end we have selected a set of points that match the spine location on the surface.

Finally the human spine has to be modeled and for that purpose we use a thirddegree polynomial. Being $\left\{P^{j}\left(x_{P^{j}}, j \lambda, z_{P^{j}}\right)\right\}$ the set of selected points for each profile, we consider the function $x_{P^{j}}=h(j \lambda)$ and we use robust linear regression [33] in order to fit $x_{P^{j}}$ as a function of $j \lambda$. Algorithm used is an iteratively re weighted least squares approach [34]. This method assign lower weight to points that do not fit well, resulting in a fit less sensitive to outliers in the input data. In this manner we have obtained a 2D curve that reflet lateral deviations of backbone. In order to obtain a $3 \mathrm{D}$ curve we project the $2 \mathrm{D}$ curve over the surface.

\section{Results}

Several 3D models of patients with spine deformities, consisting of triangular meshes, have been taken using a commercial laser scanner, model FastSCAN by Polhemus. The size of the models is about 26000 vertices and 67000 triangles. The proposed approach to obtain an estimation of the spine was applied to the models. First, the principal curvatures directions were estimated using the modified $N V V$ algorithm presented in Section 3 . Next, horizontal profiles were 

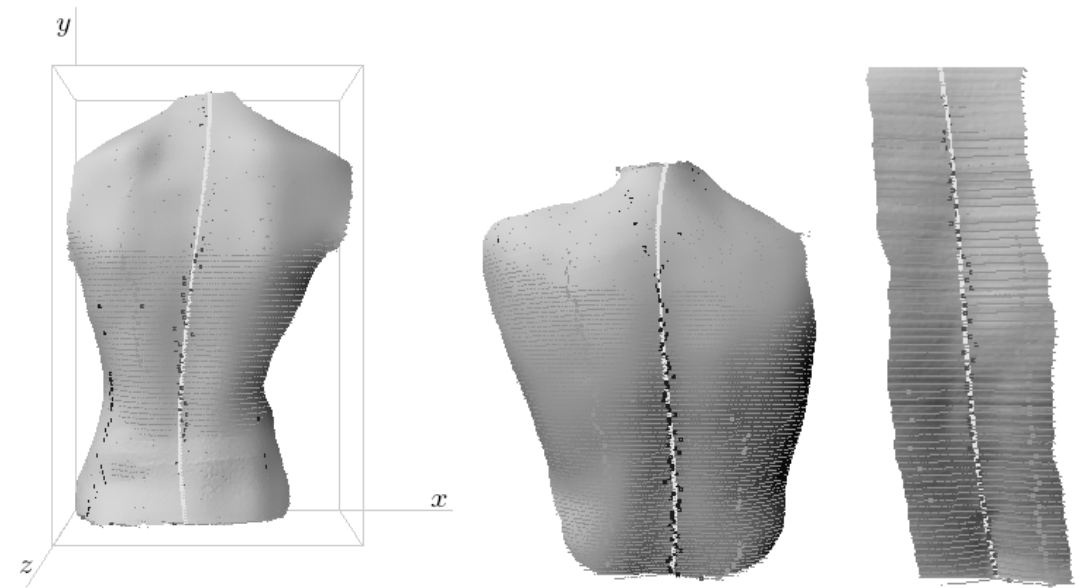

Fig. 2. Horizontal profiles, points of maximum symmetry, and curves modeling the spine in three models of real human backs

defined all along the back using the most horizontal direction of the curvatures. The mesh vertices were projected on the closest profile, and a clustering was done to obtain points all along each profile. Concave and convex regions were bounded in each profile and the best symmetry region was selected according to the concave-convex changes. Then, in each best symmetry region, and for each profile, the best symmetry point was identified. Having one best symmetry point in each profile, a three-degree polynomial curve was fit by robust least squares. Figure 2 shows the curves that model the spines estimated from three example models of real patients.

\section{Conclusions and Further Work}

We have presented a new method for estimating and modeling the human spine from scanned 3D models of the human back consisting of triangular meshes. The method is based on estimating the principal curvature directions, defining point profiles in horizontal directions, and detecting valley points. The method has been applied to real scanned human backs producing encouraging results. From the spine model important geometric information can be derived, like the Cobb angles. Further work is addressed at estimating and quantifying asymmetries in the spine and in the whole back.

\section{Acknowledgments}

This work is partially supported by the Spanish Ministry of Science and Technology under Project TIC2003-06953, and by Fundació Caixa Castelló under project P1-1B2004-08. 


\section{References}

1. Archer, I.A., Dickson, R.A. Stature and idiopathic scoliosis. A prospective study. Journal of Bone Joint Surg, Vol. 67 (1985) 185-188

2. Frobin, W., Hierholzer, E. Rasterstereography: a photogrametric method for measurement of body surfaces. Photogrammetric Engineering and Remote Sensing, Vol. 47 (1982) 1717-1724

3. Buendía, M., Salvador, R., Cibrian, R., Laguía, M., Sotoca, J.M. Determination of the object surface function by structured light: applications to the study of spinal deformities. Med. Phys. Biol., No. 44, 75-86 (1999)

4. Takasaki, H. Moiré Topography. Applied Optics, Vol. 9 (1970) 1467-1472

5. Turner-Smith, A.R. A Television/Computer three-dimensional surface shape measurement system. Journal of biomechanics, Vol. 21 (1988) 515-529

6. Drerup, B., Hierholzer, E. Automatic localization of anatomical landmarks on the back surface and construction of a body-fixed coordinate system. Journal of Biomechanics, Vol. 20, No. 10 (1987) 961-970

7. Frobin, W., Hierholzer, E. Analysis of human back shape using surface curvatures. Journal of Biomechanics, No. 15 (1982) 379-390

8. Sotoca, J.M., Buendía, M., Iñesta, J.M., Ferri, F.J. Geometric properties of the 3D spine curve. Lecture Notes in Computer Science 2652, F. J. Perales et al (eds.), Springer-Verlag, 1003-1011 (2003)

9. Cootes, T.F., Taylor, C.J., Cooper, D.H., Graham, J. Active shape models: Their Training and application. Computer vision and image understanding, No. 61, 38-59 (1995)

10. do Carmo, M. P. Differential Geometry of Curves and Surfaces. Prentice-Hall, Englewood Cliffs, NJ, (1976)

11. ONeill, B. Elementrary Differential Geometry. 2nd ed., Academic Press, Orlando, FL, (1997)

12. Besl, P.J., Jain, R.C. Invariant surface characteristics for 3d object recognition in range images. Computer Vision, Graphics, and Image Processing, No. 33, (1986) $33-80$

13. Brady, M., Ponce, J., Yuille, A., Asada, H. Describing surfaces. Computer Vision, Graphics, and Image Processing, No. 32, 1-28 (1985)

14. Feldmar, J., Ayache, N. Rigid and affine registration of smooth surfaces using differential properties. In Jan-Olof Eklundh, editor, Proceedings, European Conference on Computer Vision, number 801 in Lecture Notes in Computer Science, Springer-Verlag, 397-406 (1994)

15. Medioni, G., Nevatia, R. Description of 3-D surfaces using curvature properties In Proceedings, DARPA Image Understanding Workshop, New Orleans, LA, 291-299 (1984)

16. Guéziec, A., Ayache, N. Smoothing and matching of 3d space curves. International Journal of Computer Vision, No. 12, 79-104 (1994)

17. Monga, O., Benayoun, S., Faugeras, O. From partial derivatives of 3d density images to ridge lines. In Proceedings, IEEE Conference on Computer Vision and Pattern Recognition, 354-359 (1992)

18. Chen, X., Schmitt, F. Intrinsic surface properties from surface triangulation. In Proceedings of the European Conference on Computer Vision, Santa Margherita Ligure, Italy, 739-743 (1992)

19. Page, D. L., Sun, Y., Koschan, A.F., Paik, J., Abidi, A. Normal Vector Voting: Crease detection and curvature estimation on large, noisy meshes. Journal of Graphical Models, Vol. 64 (2003) 1-31 
20. Hagen, H., Heinz, S., Thesing, M., Schreiber, T. Simulation based modeling. Int. J. Shape Modeling, No. 4, 143-164 (1998)

21. Rössl, C., Kobbelt, L., Seidel, H. Extraction of feature lines on triangulated surfaces using morphological operators. Smart Graphics (AAAI Symposium 2000), Menlo Park, CA, AAAI Press, New York, 71-75 (2000)

22. C. Lin and M. J. Perry Shape description using surface triangulation. Proceedings of the IEEE Workshop on Computer Vision: Representation and Control, 38-43 (1982)

23. Martin, R.R. Estimation of principal curvatures from range data Int. J. Shape Modeling, No. 4, 99-109 (1998)

24. Tookey, R.M., Ball, A.A. Estimation of curvatures from planar point data. The Mathematics of Surfaces VII, Information Geometers, Winchester, 131-141 (1997)

25. Várady, T., Hermann, T. Best fit surface curvature at vertices of topologically irregular curve networks The Mathematics of Surfaces VI, Oxford University Press, Oxford, 411-427 (1996)

26. Tang, C.K., Medioni, G. Robust estimation of curvature information from noisy 3D data for shape description. Proceedings of the Seventh International Conference on Computer Vision, Kerkyra, Greece, 426-433 (1999)

27. Taubin, G. Estimating the tensor of curvature of a surface from a polyhedral approximation. Proceedings of the Fifth International Conference on Computer Vision, 902-907 (1995)

28. Meyer, M., Desbrun, M., Schroder, P., Barr, A.H. Discrete Differential-Geometry Operators for Triangulated 2-Manifolds. VisMath '02, Berlin, Germany, (2002)

29. Mitchell, J.S.B., Mount, D.M., Papadimitriou, C.H. The discrete geodesic problem. SIAM J. Comput. 16, 647-668 (1987)

30. Kimmel, R., Sethian, J.A. Computing geodesic paths on manifolds. Proc. National Academy of Sciences, 8431-8435 (1998)

31. Sun, Y., Abidi, M.A. Surface matching by 3D points fingerprint. Proceedings of the Eighth International Conference On Computer Vision, Vol. 2 (2001) 263-269

32. Hill, D. R. A vector clustering technique. In Samuelson (ed.), Mechanized Information Storage, Retrieval and Dissemination, North-Holland, Amsterdam (1968)

33. Street, J.O., Carroll, R.J., Ruppert, D. A Note on Computing Robust Regression Estimates via Iteratively Reweighted Least Squares. The American Statistician, No. 42, 152-154 (1988)

34. Holland, P.W., Welsch, R.E. Robust Regression Using Iteratively Reweighted Least-Squares. Communications in Statistics: Theory and Methods, A6, 813-827 (1977) 